CQUeST-2009-0310

\title{
Conductivity and Diffusion Constant in Lifshitz Backgrounds
}

\author{
Da-Wei Pang ${ }^{\dagger}$ \\ † Center for Quantum Spacetime, Sogang University \\ Seoul 121-742, Korea \\ pangdw@sogang.ac.kr
}

\begin{abstract}
We study the DC conductivity and the diffusion constant for asymptotically Lifshitz black branes in $(d+2)$ - dimensions with arbitrary dynamical exponent $z$. For a solvable example with $z=2, d=4$, we calculate the real-time correlation functions, from which we can read off the corresponding conductivity and diffusion constant. For black branes with arbitrary $z$ and $d$, we work out the conductivity and obtain the diffusion constant by making use of the Einstein relation. All the results agree with those obtained via the membrane paradigm.
\end{abstract}




\section{Contents}

1 Introduction

2 Asymptotically Lifshitz black branes and the membrane paradigm 3

3 Charge susceptibility and conductivity 6

4 A tractable example: $z=2, d=4 \quad 7$

5 Conductivity and diffusion constant for arbitrary $z$ and $d \quad 12$

6 Summary and discussion 14

\begin{tabular}{l|l} 
References & 16 \\
\hline
\end{tabular}

\section{Introduction}

Inspired by condensed matter physics, the investigations on non-relativistic AdS/CFT correspondence have been accelerated enormously. Such investigations may open a new window for studying physical systems in the real world, as many condensed matter systems are strongly coupled, which can be dealt with in the dual weakly coupled gravity side via the AdS/CFT correspondence. Several nice reviews are given in [1].

In many condensed matter systems near a critical point, there exist field theories with anisotropic scaling symmetry, which means that the temporal and spatial coordinates scale in different ways,

$$
t \rightarrow \lambda^{z} t, \quad x^{i} \rightarrow \lambda x^{i}
$$

where $z$ is called the 'dynamical exponent'. By now, there have been two main concrete examples of the gravity duals of non-relativistic field theories. One is the Schrödinger case, which was proposed in [2, 3] and the finite temperature generalizations were investigated in [4, 5, 6]. The other is the Lifshitz case, which was obtained in [7]. For general $(d+2)$ - 
dimensional spacetime, the dual geometry of Lifshitz fixed points is given by

$$
d s^{2}=L^{2}\left(-r^{2 z} d t^{2}+\frac{d r^{2}}{r^{2}}+r^{2} d \vec{x}^{2}\right),
$$

where $d \vec{x}^{2}=d x_{1}^{2}+\cdots+d x_{d}^{2}$. The scale transformation acts as

$$
t \rightarrow \lambda^{z} t, \quad x \rightarrow \lambda x, \quad r \rightarrow \frac{r}{\lambda}
$$

When $z=1$, it reduces to the usual AdS metric.

Unlike the Schrödinger case, it is difficult to obtain analytic black hole solutions in Lifshitz spacetimes. Four dimensional black hole solutions with $z=2$ were investigated in [8] via numerical methods. Lifshitz topological holes were studied in [9] where exact solutions were found in certain specific examples. Black holes in asymptotically Lifshitz spacetimes with arbitrary critical exponent and the corresponding thermodynamic behavior were studied in [10, 11]. Another model of non-relativistic holography was proposed in [12] where exact Lifshitz black hole solutions were obtained. Some properties of such Lifshitz black holes were studied in [14. Furthermore, certain string theory duals of Lifshitz-like fixed points were invesitigated in [15] and some no-go theorems for string duals of nonrelativistic Lifshitz-like theories were proposed in [16]. For other recent work on Lifshitz black holes see [17]. It should be emphasized that the Lifshitz black branes arise as the near-horizon geometry of certain holographic superconductors [18] as well as of charged dilaton black branes in $A d S_{4}$ [19]. Distinct string theory realizations of Lifshitz geometries were proposed quite recently in [20], where a holographic model building approach to 'strange metallic' phenomenology was initiated.

In this paper we focus on the DC conductivity and diffusion constant in Lifshitz black brane backgrounds, based on the solution obtained in [12] 1. For the relativistic counterparts, it has been verified in [21] that the electrical conductivity and charge susceptibility of a class of critical models in $d \geq 2+1$ dimensions are fixed by the central charge in a universal manner. The Weyl corrections to the conductivity and the diffusion constant were studied in [22]. For the Lifshitz case, a convenient way to calculate the diffusion constant is to apply the formula derived from the membrane paradigm [23, 24]. However, when we apply such a formula to calculate the ratio of shear viscosity over entropy density $\eta / s$, the result has a non-trivial dependence on the dynamical exponent $z$. When $z=1$, it reproduces the well-known result $1 / 4 \pi$.

\footnotetext{
${ }^{1}$ Such a solution was firstly obtained in $[13$.
} 
Therefore, one may wonder that if we can still apply the formula derived from the membrane paradigm to calculate the diffusion constant in Lifshitz backgrounds. To answer this question, we obtain the retarded Minkowskian correlation functions for a specific example $z=2, d=4$, following the standard approach [25, 26, 27]. The reason why we choose this example is simply that it is tractable. For arbitrary parameters $z$ and $d$, it is quite difficult to solve the corresponding differential equations to compute the retarded Minkowskian correlation functions. Instead we work out the DC conductivity first and obtain the diffusion constant by making use of the Einstein relation $D=\sigma / \chi$, where $\chi$ is the charge susceptibility. Our results exhibit precise agreement with those obtained via the membrane paradigm approach.

The remaining part of the paper is organized as follows: In Section 2 we review the basic properties of the Lifshitz black branes and the formulae derived via the membrane paradigm. In Section 3 we calculate the charge susceptibility $\chi$ for arbitrary $z$ and $d$ and discuss the definition of electric conductivity. In Section 4 we calculate the Minkowskian correlation functions for a specific example $z=2, d=4$ and we obtain the diffusion constant and DC conductivity which can be read off from the corresponding correlators. In Section 5 we consider the general case with arbitrary $z$ and $d$ and obtain the DC conductivity first, while the diffusion constant can be derived by the Einstein relation. All the results agree with those calculated via the membrane paradigm approach. Summary and discussion will be given in the final section.

\section{Asymptotically Lifshitz black branes and the mem- brane paradigm}

In this section we provide some backgrounds which are necessary for further discussions. It has been observed in [12] that the effective action in $(d+2)$-dimensional spacetime

$$
S=\frac{1}{16 \pi G_{d+2}} \int d^{d+2} x \sqrt{-g}\left[R-2 \Lambda-\frac{1}{2} \partial_{\mu} \phi \partial^{\mu} \phi-\frac{1}{4} e^{\lambda \phi} \mathcal{F}_{\mu \nu} \mathcal{F}^{\mu \nu}\right],
$$

where $\Lambda$ is the cosmological constant and the matter fields are a massless scalar and an abelian gauge field, admits the following Lifshitz black branes with arbitrary $z$ as solutions 
to the equations of motion

$$
\begin{aligned}
& d s^{2}=L^{2}\left[-r^{2 z} f(r) d t^{2}+\frac{d r^{2}}{r^{2} f(r)}+r^{2} \sum_{i=1}^{d} d x_{i}^{2}\right], \\
& f(r)=1-\frac{r_{0}^{z+d}}{r^{z+d}}, \quad e^{\lambda \phi}=r^{-2 d}, \quad \lambda^{2}=\frac{2 d}{z-1}, \\
& \mathcal{F}_{r t}=q_{0} r^{z+d-1}, \quad q_{0}^{2}=2 L^{2}(z-1)(z+d), \\
& \Lambda=-\frac{(z+d-1)(z+d)}{2 L^{2}},
\end{aligned}
$$

where the horizon locates at $r=r_{0}$. The temperature and entropy are given by

$$
T=\frac{z+d}{4 \pi} r_{0}^{z}, \quad S_{\mathrm{BH}}=\frac{L^{d} V_{d}}{4 G_{d+2}} r_{0}^{d}
$$

where $V_{d}$ denotes the volume of the $d$-dimensional spatial directions. When $f(r)=1$ with other field configurations remaining invariant, the zero temperature background is still a solution to the equations of motion. However, due to the non-trivial profile of the scalar field, it cannot be seen as the dual gravity description of the Lifshitz fixed points. When $z=1$, the solution turns out to be

$$
\begin{aligned}
& d s^{2}=L^{2}\left[-r^{2} f(r) d t^{2}+\frac{d r^{2}}{r^{2} f(r)}+r^{2} \sum_{i=1}^{d} d x_{i}^{2}\right] \\
& f(r)=1-\frac{r_{0}^{d+1}}{r^{d+1}}, \quad \phi=\phi_{0}=\mathrm{const}, \\
& \mathcal{F}_{r t}=0, \quad \Lambda=-\frac{d(d+1)}{2 L^{2}}
\end{aligned}
$$

which is just the ordinary Schwarzschild-AdS black brane solution. Therefore the solution cannot be treated as charged under the gauge field $\mathcal{F}_{r t}$. The scalar field $\phi$ and the gauge field $\mathcal{F}_{r t}$ simply play the role of modifying the asymptotic geometry from AdS to Lifshitz. For general $(p+2)$-dimensional metric

$$
d s^{2}=g_{00}(r) d t^{2}+g_{r r}(r) d r^{2}+g_{x x}(r) \sum_{i=1}^{p} d x_{i}^{2},
$$

the charge diffusion constant is given by the following formula

$$
D=\frac{\sqrt{-g\left(r_{0}\right)}}{g_{x x}\left(r_{0}\right) \sqrt{-g_{00}\left(r_{0}\right) g_{r r}\left(r_{0}\right)}} \int_{r_{0}}^{\infty} d r \frac{-g_{00}(r) g_{r r}(r)}{\sqrt{-g(r)}}
$$


which is derived from the membrane paradigm [23, 24]. Substituting the metric in (2.2) into (2.6), we obtain

$$
D=\frac{1}{d-z} r_{0}^{z-2}
$$

where we have assumed that $z<d$ to ensure the convergence of the integral. We can rewrite $D$ in terms of the temperature via (2.3)

$$
D=\frac{1}{d-z}\left(\frac{4 \pi}{z+d}\right)^{1-\frac{2}{z}} T^{1-\frac{2}{z}}
$$

It turns out that the charge diffusion constant $D$ has a non-trivial dependence on both the temperature $T$ and the dynamical exponent $z$. In particular, when $z=1$, the above result reduces to

$$
D=\frac{1}{4 \pi T} \frac{d+1}{d-1}
$$

which agrees with the result obtained in 21 .

However, for gravitational perturbations, the shear mode damping constant $\mathcal{D}$ is given by [23, 24]

$$
\mathcal{D}=\frac{\sqrt{-g\left(r_{0}\right)}}{\sqrt{-g_{00}\left(r_{0}\right) g_{r r}\left(r_{0}\right)}} \int_{r_{0}}^{\infty} d r \frac{-g_{00}(r) g_{r r}(r)}{g_{x x}(r) \sqrt{-g(r)}} .
$$

Note that the shear mode damping constant $\mathcal{D}=\eta /(\epsilon+P)$, where $\eta, \epsilon$ and $P$ denote the shear viscosity, the energy density and the pressure respectively. We can arrive at the following formula for $\eta / s$ by using the first law of thermodynamics $\epsilon+P=T s$

$$
\frac{\eta}{s}=T \frac{\sqrt{-g\left(r_{0}\right)}}{\sqrt{-g_{00}\left(r_{0}\right) g_{r r}\left(r_{0}\right)}} \int_{r_{0}}^{\infty} d r \frac{-g_{00}(r) g_{r r}(r)}{g_{x x}(r) \sqrt{-g(r)}} .
$$

Substituting the metric in (2.2) and the temperature in (2.3), we obtain

$$
\frac{\eta}{s}=\frac{1}{4 \pi} \frac{z+d}{d+2-z} r_{0}^{2 z-2}
$$

where the integral is convergent if we still assume $z<d$.

Although the above result can reproduce the well known KSS bound $1 / 4 \pi$ when $z=1$, the non-trivial dependence on $r_{0}$ for general $z \neq 1$ looks curious. Furthermore, it has been verified in [14] that $\eta / s$ is still $1 / 4 \pi$ for such Lifshitz black branes. This discrepancy may lead one to suspect the validity of (2.6) for Lifshitz black branes. In the subsequent sections we will see that (2.6) is still valid for Lifshitz black branes with general $z$ and $d$. 


\section{Charge susceptibility and conductivity}

In the context of relativistic AdS/CFT correspondence, an equilibrium state at finite temperature and charge density is described by the Reissner-Nördstrom-AdS black holes. For the Lifshitz case, by now we still do not know the charged solutions which are dual to equilibrium states with finite temperature and density. However, to find the charge susceptibility, we need the relation between the charge density $\rho$ and the chemical potential $\mu$ to linear order in $\mu$. Then it is sufficient to choose the uncharged Lifshitz black brane (2.2) as the background and to treat the Maxwell action

$$
S_{F}=-\frac{1}{4 g_{d+2}^{2}} \int d^{d+2} x \sqrt{-g} F_{\mu \nu} F^{\mu \nu}
$$

as perturbations. Here $g_{d+2}$ denotes the coupling constant. The Maxwell equation

$$
\frac{1}{\sqrt{-g}} \partial_{\mu}\left(\sqrt{-g} F^{\mu \nu}\right)=0
$$

gives

$$
F_{r t}=\frac{1}{r^{d+1-z}}
$$

In order to make sure that the gauge potential $A_{t} \rightarrow 0$ as $r \rightarrow \infty$, we require

$$
z-d \leq-1
$$

Notice that this condition also ensures that the integrals in (2.6) and (2.10) are convergent.

The background gauge field is given by

$$
A_{t}=\mu-\frac{C}{r^{d-z}},
$$

where $\mu$ denotes the chemical potential and the constant $C$ is related to the charge density. The chemical potential $\mu$ is fixed by the condition that $A_{t}$ vanishes at the horizon $r=r_{0}$, which gives

$$
C=\mu r_{0}^{d-z}
$$

The charge density $\rho$ can be determined by

$$
\rho=\frac{\delta \mathcal{L}}{\delta\left(\partial_{r} A_{t}\right)}=\frac{L^{d-2}}{g_{d+2}^{2}}(d-z) \mu r_{0}^{d-z} .
$$


Finally, the charge susceptibility is fixed by the formula $\rho=\chi \mu$,

$$
\chi=\frac{L^{d-2}}{g_{d+2}^{2}}(d-z) r_{0}^{d-z} .
$$

We shall define the electrical conductivity following the approach proposed in [21]. Since the dual gravity descriptions typically do not have dynamical $U(1)$ gauge fields, we have to clarify the meaning of conductivity first. We imagine gauging a global $U(1)$ symmetry of the theory with a small coupling $e$, and work to leading order in $e$. The electrical conductivity is defined with respect to this $U(1)$ gauge field. At leading order, the effects of the gauge field can be neglected and the electromagnetic response can be determined from the original theory. Then the conserved current $J_{\mu}$ of the $U(1)$ gauge field is rescaled as $J_{\mu} \rightarrow e J_{\mu}$ and a factor of $e^{2}$ will appear in both the susceptibility and the conductivity. Finally, the DC conductivity is defined by

$$
\sigma=-e^{2} \lim _{\omega \rightarrow 0} \frac{1}{\omega} \operatorname{Im} G_{i i}^{R}(\omega, \mathbf{q}=0),
$$

where $G_{i i}^{R}(\omega, \mathbf{q})$ is the retarded real-time current-current correlation function. For simplicity, we will set $e^{2}=1$ in the subsequent discussions.

\section{A tractable example: $z=2, d=4$}

In this section we study a concrete example, i.e. $z=2, d=4$ Lifshitz black brane, following the standard procedures proposed in [26, 27]. The main reason why we study this example is simply that it is tractable. We have known in Section 2 that $z$ and $d$ should satisfy $z-d \leq-1$ such that the gauge potential has an appropriate asymptotic behavior. Thus in four dimensions $z>1$ is not allowed while in five dimensions the corresponding differential equations are difficult to solve. We will obtain the retarded Minkowskian correlation functions for $z=2, d=4$ case and read off the charge diffusion constant and DC conductivity from the correlation functions. The DC conductivity can also be determined from the real-time current-current correlation function and the charge diffusion constant can be obtained via the Einstein relation $D=\sigma / \chi$. We will find that the results given by these two approaches agree.

The main steps for calculating retarded Minkowskian correlation functions can be summarized as follows [25, 26]: 
(1) Extracting the function $B(u)$ staying in front of the kinetic term $\left(\partial_{\mu} \phi\right)^{2}$ from the action for a certain field $\phi$,

$$
S=\frac{1}{2} \int d u d^{d+1} x B(u)\left(\partial_{u} \phi\right)^{2}+\cdots
$$

where $u$ denotes the radial coordinate and the boundary locates at $u=0$.

(2) Solving the linearized equation for $\phi$ and expressing the bulk field $\phi$ via its boundary value $\phi_{0}$,

$$
\phi(u, q)=f_{q}(u) \phi_{0}(q)
$$

In Minkowski space one has to specify the boundary condition at the horizon as well as that at the boundary $u=0$. We choose the incoming-wave boundary condition for all Fourier components $\phi_{q}$ with timelike $q$ and require regularity for spacelike q's.

(3) Then the retarded Minkowskian correlation function is

$$
G^{R}(q)=\left.B(u) f_{-q}(u) \partial_{u} f_{q}(u)\right|_{u=0}
$$

Let us rewrite the six-dimensional $z=2$ Lifshitz black brane as

$$
d s^{2}=L^{2}\left[-r^{4} f(r) d t^{2}+\frac{d r^{2}}{r^{2} f(r)}+r^{2} \sum_{i=1}^{4} d x_{i}^{2}\right], \quad f(r)=1-\frac{r_{0}^{6}}{r^{6}},
$$

where the Hawking temperature is given by

$$
T=\frac{6 r_{0}^{2}}{4 \pi}
$$

Introducing the new radial coordinate $u=r_{0}^{2} / r^{2}$, the black brane metric turns out to be

$$
d s^{2}=L^{2}\left[-\frac{r_{0}^{4}}{u^{2}} f(u) d t^{2}+\frac{d u^{2}}{4 u^{2} f(u)}+\frac{r_{0}^{2}}{u} \sum_{i=1}^{4} d x_{i}^{2}\right], \quad f(u)=1-u^{3},
$$

where the boundary locates at $u=0$ and the horizon locates at $u=1$.

The subsequent calculations closely follow [26]. We choose the radial gauge $A_{u}=0$ and Fourier decomposition

$$
A_{\mu}=\int \frac{d^{d+1} q}{(2 \pi)^{d+1}} e^{-i \omega t+i \mathbf{q} \cdot \mathbf{x}} A_{\mu}(q, u)
$$


We further fix the spatial momentum by $q_{4}=q$ and $q_{\beta}=0$ for $\beta=1,2,3$. The Maxwell equations $1 / \sqrt{-g} \partial_{\mu}\left(\sqrt{-g} F^{\mu \nu}\right)=0$ can be rewritten as

$$
\begin{gathered}
A_{t}^{\prime \prime}-\frac{1}{4 r_{0}^{2} u f(u)}\left(\omega q A_{x}+q^{2} A_{t}\right)=0, \\
\omega A_{t}^{\prime}+q r_{0}^{2} \frac{f(u)}{u} A_{x}^{\prime}=0, \\
\partial_{u}\left(\frac{f(u)}{u} A_{x}^{\prime}\right)+\frac{1}{4 r_{0}^{4} u f(u)}\left(\omega^{2} A_{x}+\omega q A_{t}\right)=0, \\
\partial_{u}\left(\frac{f(u)}{u} A_{\beta}^{\prime}\right)+\frac{\omega^{2}}{4 r_{0}^{4} u f(u)} A_{\beta}-\frac{q^{2}}{4 r_{0}^{2} u^{2}} A_{\beta}=0,
\end{gathered}
$$

where we have denoted $x_{4} \equiv x$ and the prime stands for derivative with respect to $u$. Notice that all the equations are not independent, as we can obtain (4.10) by combining (4.8) and (4.9).

The following calculations are straightforward. First we can arrive at the following equation for $A_{t}$ via (4.8) and (4.9)

$$
A_{t}^{\prime \prime \prime}+\frac{f^{\prime}(u)}{f(u)} A_{t}^{\prime \prime}+\frac{1}{u} A_{t}^{\prime \prime}+\frac{\omega^{2}}{4 r_{0}^{4} f^{2}(u)} A_{t}^{\prime}-\frac{q^{2}}{4 r_{0}^{2} u f(u)} A_{t}^{\prime}=0 .
$$

To determine the behavior of $A_{t}^{\prime}$ near the singular point $u=1$, we set $A_{t}^{\prime}=(1-u)^{\alpha} F(u)$, where $F(u)$ is a regular function and substitute it into (4.12). One can find that $\alpha^{2}=$ $-\omega^{2} / 36 r_{0}^{4}$ and the incoming-wave boundary condition determines

$$
\alpha=-\frac{i \omega}{6 r_{0}^{2}}=-\frac{i \omega}{4 \pi T}
$$

Next we will solve the differential equation perturbatively by setting

$$
F(u)=F_{0}(u)+\omega F_{1}(u)+q^{2} G_{1}(u)+\mathcal{O}\left(\omega^{2}, \omega q, q^{2}\right) .
$$

The equation for $F_{0}(u)$ is given by

$$
F_{0}^{\prime \prime}(u)+\frac{f^{\prime}(u)}{f(u)} F_{0}^{\prime}(u)+\frac{1}{u} F_{0}^{\prime}(u)=0,
$$

whose solution is simply $F_{0}(u)=C$, where $C$ is a constant. Then the equations for $F_{1}(u)$ and $G_{1}(u)$ can be simplified considerably,

$$
F_{1}^{\prime \prime}(u)+\left[\frac{f^{\prime}(u)}{f(u)}+\frac{1}{u}\right] F_{1}^{\prime}(u)+\frac{i C}{4 \pi T}\left[\frac{f^{\prime}(u)}{(1-u) f(u)}+\frac{1}{u(1-u)^{2}}\right]=0,
$$




$$
G_{1}^{\prime \prime}(u)+\left[\frac{f^{\prime}(u)}{f(u)}+\frac{1}{u}\right] G_{1}^{\prime}(u)-\frac{C}{4 r_{0}^{2} u f(u)}=0 .
$$

The solutions are given by

$$
\begin{gathered}
F_{1}(u)=\frac{i C}{4 \pi T}\left[3 \ln u-\ln \frac{1}{3}\left(1+u+u^{2}\right)\right], \\
G_{1}(u)=\frac{C}{4 r_{0}^{2}}\left[-\ln u+\frac{1}{2} \ln \frac{1}{3}\left(1+u+u^{2}\right)+\frac{\sqrt{3}}{3}\left(\tan ^{-1} \frac{2 u+1}{\sqrt{3}}-\frac{\pi}{3}\right)\right],
\end{gathered}
$$

where we have fixed the integration constants by requiring that $F_{1}(u), G_{1}(u)$ are regular at the horizon $u=1$ and $F_{1}(1)=G_{1}(1)=0$.

We can also obtain the following relation between $A_{x}$ and $A_{t}$ from (4.8),

$$
A_{x}=\frac{4 r_{0}^{2} u f(u)}{\omega q} A_{t}^{\prime \prime}-\frac{q}{\omega} A_{t} .
$$

Then the integration constant $C$ can be fixed in terms of the boundary value of the fields $A_{t}^{0}, A_{x}^{0}$,

$$
C=\frac{q \omega A_{x}^{0}+q^{2} A_{t}^{0}}{2 i \omega-q^{2}} .
$$

We will see that the pole in $C$ is the same pole that appears in the retarded Green's functions.

The Maxwell action can be recast as

$$
\begin{aligned}
S_{F} & =-\frac{1}{2 g_{6}^{2}} \int d u d^{5} x \sqrt{-g} g^{u u} g^{i j} \partial_{u} A_{i} \partial_{u} A_{j} \\
& =\frac{L^{2} r_{0}^{2}}{g_{6}^{2}} \int d u d^{5} x\left[A_{t}^{\prime 2}-r_{0}^{2} \frac{f(u)}{u} A_{i}^{\prime 2}\right] .
\end{aligned}
$$

Then we can extract the function $B(u)$,

$$
B_{t}(u)=\frac{2 L^{2} r_{0}^{2}}{g_{6}^{2}}, \quad B_{i}(u)=-\frac{2 L^{2} r_{0}^{4}}{g_{6}^{2}} \frac{f(u)}{u}
$$

We can easily obtain the real-time correlation functions following the standard prescriptions

$$
\begin{aligned}
G_{t t}^{R} & =\frac{L^{2} r_{0}^{2}}{g_{6}^{2}} \frac{q^{2}}{i \omega-\frac{1}{2} q^{2}}, \\
G_{x x}^{R} & =\frac{L^{2} r_{0}^{2}}{g_{6}^{2}} \frac{\omega^{2}}{i \omega-\frac{1}{2} q^{2}},
\end{aligned}
$$




$$
G_{t x}^{R}=G_{x t}^{R}=-\frac{L^{2} r_{0}^{2}}{g_{6}^{2}} \frac{\omega q}{i \omega-\frac{1}{2} q^{2}} .
$$

By making use of the general form of the retarded correlation function

$$
G_{t t}^{R}=\frac{\chi D q^{2}}{i \omega-D q^{2}}
$$

and the Einstein relation $\sigma=\chi D$, we can read off the diffusion constant and the conductivity

$$
D=\frac{1}{2}, \quad \sigma=\frac{L^{2} r_{0}^{2}}{g_{6}^{2}}
$$

Comparing these results with (2.7), we can find precise agreement when $z=2, d=4$.

Unfortunately, the differential equation for $A_{\beta}$ is difficult to solve, so we cannot obtain the corresponding retarded correlation functions. However, to obtain the conductivity we just need to calculate $G_{\beta \beta}^{R}(\omega, q=0)$. Then the equation for $A_{\beta}$ is given by

$$
\partial_{u}\left(\frac{f(u)}{u} A_{\beta}^{\prime}\right)+\frac{\omega^{2}}{4 r_{0}^{4} u f(u)} A_{\beta}=0
$$

Similarly, we assume $A_{\beta}=(1-u)^{\gamma} H(u)$, where $H(u)$ is a regular function. The incomingwave boundary condition still forces us to choose $\gamma=-i \omega / 4 \pi T$. Next we solve for $H(u)$ perturbatively,

$$
H(u)=H_{0}(u)+\omega H_{1}(u)+\mathcal{O}\left(\omega^{2}\right) .
$$

The equation for $H_{0}$ is given by

$$
H_{0}^{\prime \prime}(u)+\frac{f^{\prime}(u)}{f(u)} H_{0}^{\prime}(u)-\frac{1}{u} H_{0}^{\prime}(u)=0,
$$

and the solution is simply

$$
H_{0}(u)=H_{0}=\text { const. }
$$

The equation for $H_{1}(u)$ takes the following simple form after fixing $H_{0}$,

$$
H_{1}^{\prime \prime}(u)+\left[\frac{f^{\prime}(u)}{f(u)}-\frac{1}{u}\right] H_{1}^{\prime}(u)+\frac{i H_{0}}{4 \pi T}\left[\frac{f^{\prime}(u)}{(1-u) f(u)}+\frac{2 u-1}{u(1-u)^{2}}\right]=0,
$$

whose solution is

$$
H_{1}(u)=\frac{i H_{0}}{4 \pi T}\left[\frac{1}{2} \ln \frac{1}{3}\left(1+u+u^{2}\right)-\sqrt{3}\left(\tan ^{-1} \frac{2 u+1}{\sqrt{3}}-\frac{\pi}{3}\right)\right] .
$$


Here the integration constants have been fixed such that $H_{1}(u)$ is regular at the horizon $u=1$ and $H_{1}(1)=0$.

The constant $H_{0}$ is fixed by

$$
H_{0}=\frac{A_{\beta}^{0}}{1+\frac{i \omega}{4 \pi T}\left(\frac{\pi}{2 \sqrt{3}}-\ln 3\right)},
$$

where $A_{\beta}^{0}$ stands for the boundary value of $A_{\beta}$. Finally, from (4.3) we can obtain

$$
G_{\beta \beta}^{R}(\omega, q=0)=-\frac{L^{2} r_{0}^{2}}{g_{6}^{2}} i \omega
$$

Then the DC conductivity is given by

$$
\sigma=-\lim _{\omega \rightarrow 0} \frac{1}{\omega} \operatorname{Im} G_{\beta \beta}^{R}(\omega, q=0)=\frac{L^{2} r_{0}^{2}}{g_{6}^{2}} .
$$

We can determine the diffusion constant by using (3.6) and the Einstein relation,

$$
D=\frac{\sigma}{\chi}=\frac{1}{2}
$$

Therefore we find precise agreement with (2.7) for $z=2, d=4$ once again.

\section{Conductivity and diffusion constant for arbitrary $z$ and $d$}

In this section we will calculate the DC conductivity and diffusion constant for arbitrary $z$ and $d$. Generally speaking, the differential equations for arbitrary $z$ and $d$ are difficult to solve thus we can hardly read off the conductivity and diffusion constant from the retarded correlation functions straightforwardly. However, as stated in the previous section. We can obtain the conductivity first by evaluating the retarded correlation function $G_{i i}^{R}(\omega, q=$ $0)$, which is always tractable. The diffusion constant can be obtained by the Einstein relation $D=\sigma / \chi$. We will see that the results for general $z$ and $d$ agree with those obtained via the membrane paradigm.

Recall the Lifshitz black brane solution for arbitrary $z$ and $d$

$$
d s^{2}=L^{2}\left[-r^{2 z} f(r) d t^{2}+\frac{d r^{2}}{r^{2} f(r)}+r^{2} \sum_{i=1}^{d} d x_{i}^{2}\right], \quad f(r)=1-\frac{r_{0}^{z+d}}{r^{z+d}} .
$$


Consider the coordinate transformation $u=r_{0} / r$, the metric can be rewritten as

$$
d s^{2}=L^{2}\left[-\frac{r_{0}^{2 z}}{u^{2 z}} f(u) d t^{2}+\frac{d u^{2}}{u^{2} f(u)}+\frac{r_{0}^{2}}{u^{2}} \sum_{i=1}^{d} d x_{i}^{2}\right], \quad f(u)=1-u^{z+d} .
$$

The horizon still locates at $u=1$ and the boundary locates at $u=0$.

Since we only need to work out $G_{i i}^{R}(\omega, q=0)$, we can assume the gauge field to be $A_{i}=A_{i}(u) e^{-i \omega t}$. The corresponding Maxwell equations can be written as

$$
\partial_{u}\left(\frac{f(u)}{u^{z+d-3}} A_{i}^{\prime}(u)\right)+\omega^{2} \frac{u^{z-d+1}}{r_{0}^{2 z} f(u)} A_{i}(u)=0
$$

where the prime denotes derivative with respect to the radial coordinate $u$. Let $A_{i}(u)=$ $(1-u)^{\delta} A(u)$, where $A(u)$ is a regular function. Similarly, the regularity at $u=1$ and the incoming-wave boundary condition still fix $\delta=-i \omega / 4 \pi T$.

Next we solve for $A(u)$ perturbatively,

$$
A(u)=A_{0}(u)+i \omega A_{1}(u)+\mathcal{O}\left(\omega^{2}\right) .
$$

The equation for $A_{0}(u)$ is given by

$$
A_{0}^{\prime \prime}(u)+\frac{f^{\prime}(u)}{f(u)} A_{0}^{\prime}(u)-\frac{z+d-3}{u} A_{0}^{\prime}(u)=0,
$$

whose solution is still simply

$$
A_{0}(u)=A_{0}=\text { const. }
$$

The equation for $A_{1}(u)$ can be written as

$$
A_{1}^{\prime \prime}(u)+\left[\frac{f^{\prime}(u)}{f(u)}-\frac{z+d-3}{u}\right] A_{1}^{\prime}(u)+\frac{A_{0}}{4 \pi T}\left[\frac{1}{(1-u)^{2}}+\frac{f^{\prime}(u)}{f(u)(1-u)}-\frac{z+d-3}{u(1-u)}\right]=0 .
$$

The solution for $A_{1}(u)$ can be expressed in terms of hypergeometric functions,

$$
A_{1}(u)=\frac{A_{0}}{r_{0}^{z}}\left(\frac{1}{2 u^{2}}-\frac{1}{2 u^{2}}{ }_{2} F_{1}\left[-\frac{2}{z+d}, 1,1-\frac{2}{z+d}, u^{z+d}\right]\right)+C_{1}-\frac{A_{0}}{4 \pi T} \ln (1-u),
$$

where we have fixed one integration constant by requiring that $A_{1}(u)$ is regular at $u=1$ and the other integration constant $C_{1}$ ensures that $A_{1}(u)$ vanishes at $u=1$. Moreover, $A_{0}$ can be determined in terms of the boundary value $A_{i}^{0}$,

$$
A_{i}^{0}=A_{0}+\mathcal{O}(\omega)
$$


Now let us rewrite the Maxwell action as

$$
\begin{aligned}
S_{F} & =-\frac{1}{2 g_{d+2}^{2}} \int d u d^{d+1} x \sqrt{-g} g^{u u} g^{i j} \partial_{u} A_{i} \partial_{u} A_{j} \\
& =-\frac{1}{2 g_{d+2}^{2}} \int d u d^{d+1} x \frac{L^{d-2} r_{0}^{z+d-2}}{u^{z+d-3}} f(u)\left(\partial_{u} A_{i}\right)^{2} .
\end{aligned}
$$

Then we can extract the function $B(u)$

$$
B(u)=-\frac{L^{d-2} r^{z+d-2}}{g_{d+2}^{2} u^{z+d-3}} f(u) .
$$

Finally, the retarded correlation function is given by

$$
G_{i i}^{R}(\omega, q=0)=-\frac{L^{d-2} r_{0}^{d-2}}{g_{d+2}^{2}} i \omega .
$$

Then the DC conductivity is determined as

$$
\sigma=-\lim _{\omega \rightarrow 0} \frac{1}{\omega} \operatorname{Im} G_{i i}^{R}(\omega, q=0)=\frac{L^{d-2} r_{0}^{d-2}}{g_{d+2}^{2}} .
$$

By combining the charge susceptibility (3.6) and the Einstein relation, we can arrive at

$$
D=\frac{1}{d-z} r_{0}^{z-2}
$$

which agrees with the result given by the membrane paradigm (2.7) for arbitrary $z$ and $d$.

\section{Summary and discussion}

The membrane paradigm provides us an efficient way of investigating the hydrodynamics of black holes. In particular, the charge diffusion constant and the ratio of shear viscosity over entropy density $\eta / s$ can be evaluated in terms of the black hole metric and temperature. However, when we apply the formula to the Lifshitz black branes straightforwardly, we find that the well-known result $\eta / s=1 / 4 \pi$ cannot be reproduced. This leads one to suspect the validity of the formula for the charge diffusion constant. In this paper we study the conductivity and the charge diffusion constant for Lifshitz black branes. We calculate the retarded correlation functions for a specific, tractable example $z=2, d=4$, following the standard approach proposed in [26]. Then we read off the charge diffusion 
constant and the conductivity from the $t$-component of the correlators. We also obtain the conductivity for general $z$ and $d$ by evaluating the corresponding correlation functions with $q=0$ and the charge diffusion constant via the Einstein relation. All these results agree with those obtained via the membrane paradigm. This verifies the validity of the formula for the charge diffusion constant derived from the membrane paradigm. However, one may need to find the appropriate formula for $\eta / s$ in the Lifshitz black brane backgrounds.

For the relativistic counterparts, it has been shown in [21] that for a large class of critical models in $d \geq 2+1$ dimensions, the electrical conductivity and charge susceptibility are fixed the central charge in a universal manner. A universal relation for $\sigma / \chi$ is proposed, which looks similar to the famous relation for $\eta / s$. It was further shown in 22] that such a lower bound still held if the Weyl corrections were considered. However, as pointed out in [21], if $\hbar$ and the speed of light $v$ were restored, the ratio turned out to be

$$
\frac{\sigma}{\chi} \geq \frac{\hbar v^{2}}{4 \pi T} \frac{d}{d-2}
$$

Such a bound cannot hold in non-relativistic systems as the speed of light $v \rightarrow \infty$. Therefore we cannot expect a similar bound for the Lifshitz black branes.

It should be pointed out that comparing the relativistic counterparts, i.e. RN-AdS black holes, we are still lack of charged black brane solutions in asymptotically Lifshitz spacetime, which can be seen as the dual gravity description of field theories at Lifshitz fixed points at finite temperature and charge density. Once we obtain such charged black brane solutions, we may investigate their thermodynamic and hydrodynamic properties, following e.g. [28, 29, 30, 31]. Moreover, three distinct string theory realizations of Lifshitz geometries were outlined in [20]. It would be interesting to study the corresponding properties of such Lifshitz geometries in the context of string theory via a top-down approach.

\section{Acknowledgements}

DWP would like to thank Rong-Gen Cai, Bom Soo Kim and Jian-Huang She for helpful discussions. This work was supported by the National Research Foundation of Korea(NRF) grant funded by the Korea government(MEST) through the Center for Quantum Spacetime(CQUeST) of Sogang University with grant number 2005-0049409. 


\section{References}

[1] S. A. Hartnoll, "Lectures on holographic methods for condensed matter physics," Class. Quant. Grav. 26, 224002 (2009) [arXiv:0903.3246 [hep-th]].

C. P. Herzog, "Lectures on Holographic Superfluidity and Superconductivity," J. Phys. A 42, 343001 (2009) [arXiv:0904.1975 [hep-th]].

J. McGreevy, "Holographic duality with a view toward many-body physics," arXiv:0909.0518 [hep-th].

[2] D. T. Son, "Toward an AdS/cold atoms correspondence: a geometric realization of the Schroedinger symmetry," Phys. Rev. D 78, 046003 (2008) arXiv:0804.3972 [hep-th]].

[3] K. Balasubramanian and J. McGreevy, "Gravity duals for non-relativistic CFTs," Phys. Rev. Lett. 101, 061601 (2008) arXiv:0804.4053 [hep-th]].

[4] C. P. Herzog, M. Rangamani and S. F. Ross, "Heating up Galilean holography," JHEP 0811, 080 (2008) [arXiv:0807.1099 [hep-th]].

[5] J. Maldacena, D. Martelli and Y. Tachikawa, "Comments on string theory backgrounds with non-relativistic conformal symmetry," JHEP 0810, 072 (2008) arXiv:0807.1100 [hep-th]].

[6] A. Adams, K. Balasubramanian and J. McGreevy, "Hot Spacetimes for Cold Atoms," JHEP 0811, 059 (2008) [arXiv:0807.1111 [hep-th]].

[7] S. Kachru, X. Liu and M. Mulligan, "Gravity Duals of Lifshitz-like Fixed Points," Phys. Rev. D 78, 106005 (2008) [arXiv:0808.1725 [hep-th]].

[8] U. H. Danielsson and L. Thorlacius, "Black holes in asymptotically Lifshitz spacetime," JHEP 0903, 070 (2009) [arXiv:0812.5088 [hep-th]].

[9] R. B. Mann, "Lifshitz Topological Black Holes," JHEP 0906, 075 (2009) arXiv:0905.1136 [hep-th]].

[10] G. Bertoldi, B. A. Burrington and A. Peet, "Black Holes in asymptotically Lifshitz spacetimes with arbitrary critical exponent," arXiv:0905.3183 [hep-th]. 
[11] G. Bertoldi, B. A. Burrington and A. W. Peet, "Thermodynamics of black branes in asymptotically Lifshitz spacetimes," arXiv:0907.4755 [hep-th].

[12] M. Taylor, "Non-relativistic holography," arXiv:0812.0530 [hep-th].

[13] P. Koroteev and M. Libanov, "On Existence of Self-Tuning Solutions in Static Braneworlds without Singularities," JHEP 0802, 104 (2008) arXiv:0712.1136 [hepth]].

[14] D. W. Pang, "A Note on Black Holes in Asymptotically Lifshitz Spacetime," arXiv:0905.2678 [hep-th].

[15] T. Azeyanagi, W. Li and T. Takayanagi, "On String Theory Duals of Lifshitz-like Fixed Points," JHEP 0906, 084 (2009) [arXiv:0905.0688 [hep-th]].

[16] W. Li, T. Nishioka and T. Takayanagi, "Some No-go Theorems for String Duals of Non-relativistic Lifshitz-like Theories," JHEP 0910, 015 (2009) arXiv:0908.0363 [hep-th]].

[17] D. W. Pang, " $R^{2}$ Corrections to Asymptotically Lifshitz Spacetimes," JHEP 0910, 031 (2009) arXiv:0908.1272 [hep-th]].

E. J. Brynjolfsson, U. H. Danielsson, L. Thorlacius and T. Zingg, "Holographic Superconductors with Lifshitz Scaling," arXiv:0908.2611 [hep-th].

K. Balasubramanian and J. McGreevy, "An analytic Lifshitz black hole," arXiv:0909.0263 [hep-th].

E. Ayon-Beato, A. Garbarz, G. Giribet and M. Hassaine, "Lifshitz Black Hole in Three Dimensions," Phys. Rev. D 80, 104029 (2009) [arXiv:0909.1347 [hep-th]].

R. G. Cai, Y. Liu and Y. W. Sun, "A Lifshitz Black Hole in Four Dimensional $R^{2}$ Gravity," JHEP 0910, 080 (2009) [arXiv:0909.2807 [hep-th]].

S. J. Sin, S. S. Xu and Y. Zhou, "Holographic Superconductor for a Lifshitz fixed point," arXiv:0909.4857 [hep-th].

Y. S. Myung, Y. W. Kim and Y. J. Park, "Dilaton gravity approach to three dimensional Lifshitz black hole," arXiv:0910.4428 [hep-th].

D. W. Pang, "On Charged Lifshitz Black Holes," arXiv:0911.2777 [hep-th].

[18] S. S. Gubser and A. Nellore, "Ground states of holographic superconductors," Phys. Rev. D 80, 105007 (2009) [arXiv:0908.1972 [hep-th]]. 
[19] K. Goldstein, S. Kachru, S. Prakash and S. P. Trivedi, "Holography of Charged Dilaton Black Holes," arXiv:0911.3586 [hep-th].

[20] S. A. Hartnoll, J. Polchinski, E. Silverstein and D. Tong, "Towards strange metallic holography," arXiv:0912.1061 [hep-th].

[21] P. Kovtun and A. Ritz, "Universal conductivity and central charges," Phys. Rev. D 78, 066009 (2008) arXiv:0806.0110 [hep-th]].

[22] A. Ritz and J. Ward, "Weyl corrections to holographic conductivity," Phys. Rev. D 79, 066003 (2009) arXiv:0811.4195 [hep-th]].

[23] P. Kovtun, D. T. Son and A. O. Starinets, "Holography and hydrodynamics: Diffusion on stretched horizons," JHEP 0310, 064 (2003) arXiv:hep-th/0309213.

[24] N. Iqbal and H. Liu, "Universality of the hydrodynamic limit in AdS/CFT and the membrane paradigm," Phys. Rev. D 79, 025023 (2009) arXiv:0809.3808 [hep-th]].

[25] D. T. Son and A. O. Starinets, "Minkowski-space correlators in AdS/CFT correspondence: Recipe and applications," JHEP 0209, 042 (2002) arXiv:hep-th/0205051].

[26] G. Policastro, D. T. Son and A. O. Starinets, "From AdS/CFT correspondence to hydrodynamics," JHEP 0209, 043 (2002) arXiv:hep-th/0205052.

[27] C. P. Herzog, "The hydrodynamics of M-theory," JHEP 0212, 026 (2002) arXiv:hep-th/0210126.

[28] C. P. Herzog, P. Kovtun, S. Sachdev and D. T. Son, "Quantum critical transport, duality, and M-theory," Phys. Rev. D 75, 085020 (2007) arXiv:hep-th/0701036.

[29] S. A. Hartnoll and P. Kovtun, "Hall conductivity from dyonic black holes," Phys. Rev. D 76, 066001 (2007) [arXiv:0704.1160 [hep-th]].

[30] S. A. Hartnoll, P. K. Kovtun, M. Muller and S. Sachdev, "Theory of the Nernst effect near quantum phase transitions in condensed matter, and in dyonic black holes," Phys. Rev. B 76, 144502 (2007) [arXiv:0706.3215 [cond-mat.str-el]].

[31] S. A. Hartnoll and C. P. Herzog, "Ohm's Law at strong coupling: S duality and the cyclotron resonance," Phys. Rev. D 76, 106012 (2007) arXiv:0706.3228 [hep-th]]. 